\title{
Fatores determinantes na contratação de um personal trainer em Fortaleza/CE
}

\author{
Determining factors in hiring a personal trainer in Fortaleza/CE/BRAZIL \\ Factores determinantes en la contratación de un personal trainer en Fortaleza/CE
}

Abraham Lincoln de Paula Rodrigues'

\begin{abstract}
Resumo
O estudo objetivou conhecer os fatores determinantes na contratação do personal trainer em uma academia de Fortaleza-CE. Foram selecionados 30 voluntários de acordo com os critérios de inclusão e exclusão do estudo. A amostra caracteriza-se como sendo formada por jovens adultas, que treinam acompanhadas $3 \mathrm{x}$ por semana, utilizando o serviço há mais de 12 meses. A metodologia de trabalho foi o principal fator apontado como determinante na contratação do personal. O nível de exigência de qualificação está cada vez maior por parte dos clientes, além do conhecimento técnico, faz-se importante o profissional desenvolver habilidades relacionadas aos tratos humanísticos e interpessoais.
\end{abstract}

Palavras-chave: Motivação; Contratação; Personal Trainer

\begin{abstract}
The study aimed to know the determining factors in the hiring of the personal trainer in a gym in Fortaleza City, in CE State, Brazil. Thirty volunteers were selected according to the inclusion and exclusion criteria of the study. The sample is characterized as being formed by young adults, who train accompanied three times a week, using the service for more than twelve months. The work methodology was the main factor identified as a determinant in hiring the personal. The level of qualification requirements is increasingly higher on the part of clients, in addition to technical knowledge, it is important for professionals to develop skills related to humanistic and interpersonal treatment.
\end{abstract}

Keywords: Motivation; Hiring; Personal Trainer

\footnotetext{
${ }^{\mathrm{I}}$ Universidade de Fortaleza - UNIFOR, Fortaleza, CE, Brasil - Endereço: Av. Washington Soares, 1321 - Edson Queiroz - Fortaleza - CE, Brasil, CEP: 60811-905 - e-mail: $\underline{\text { lincoln7777@ hotmail.com }}$
} 


\section{Resumen}

El estudio objetivó conocer los factores determinantes en la contratación del personal trainer en un gimnasio Fortaleza-CE, Brasil. Se seleccionaron 30 voluntarios de acuerdo con los criterios de inclusión y exclusión del estudio. La muestra se caracteriza como formada por jóvenes adultas, que entrenan acompañadas 3 veces por semana, utilizando el servicio desde hace más de 12 meses. La metodología de trabajo fue el principal factor señalado como determinante en la contratación del personal. El nivel de exigencia de calificación es cada vez mayor por parte de los clientes, además del conocimiento técnico, se hace importante el profesional desarrollar habilidades relacionadas a los tratos humanísticos e interpersonales.

Palabras Clave: Motivación; Contratación; Entrenador/a Personal

\section{Introdução}

Segundo Santos e Knijnik (2006), a prática de exercício físico, adequada às condições individuais e com a devida instrução, traz vários benefícios aos seus praticantes, dentre os quais se podem destacar melhorias relacionadas ao bem-estar físico, psicológico e social. Corroborando esse pensamento, Freitas et al. (2007) afirmam que o efeito benéfico de um estilo de vida ativo contribui para manutenção da capacidade funcional, da autonomia física, minimizando a degeneração provocada pelo envelhecimento.

O treinamento personalizado, prestado pelo personal trainer, apresenta-se como uma tendência atual no mercado fitness e leva a um comportamento de busca de melhoria da estética, da performance, e do bem estar, sendo que o profissional de Educação Física busca a melhora no conhecimento, para suprir as necessidades de seus clientes (BERESFORD; SILVA; GARAY, 2008; TAHARA; SILVA, 2003).

Além dos itens supracitados, existe também o aspecto motivacional, o qual poderá influenciar no sucesso da obtenção dos resultados almejados através da prática de exercício físico. Aspectos como o comprometimento com horários, a exclusividade do professor, a individualidade no treinamento e a valorização da autoestima em busca dos resultados, com segurança, fazem com que seja valorizada a aquisição do serviço de um personal trainer. (PRADO; LIBERALI, 2008).

Diante da relevância do conteúdo exposto nos parágrafos anteriores, optou-se por realizar este estudo que objetivou verificar os fatores relevantes para contratação do serviço de um personal trainer em uma academia do município de Fortaleza/CE. 


\section{Materiais e Métodos}

\subsection{Tipo e local do estudo}

Trata-se de uma pesquisa direta, de caráter descritivo, realizada de maneira transversal e com uma abordagem predominantemente quantitativa (LIBERALI, 2008). O local escolhido para a realização do estudo foi uma academia localizada no bairro Aldeota, no município de Fortaleza/CE. A escolha do local deu-se por conta da facilidade de acesso do autor, além do local reunir um número considerável de indivíduos que realizam seus treinamentos com personal trainer, o que possibilitou a captação de uma amostra maior.

\subsection{Sujeitos do estudo}

$\mathrm{O}$ universo da pesquisa contemplou indivíduos que treinavam com personal trainer no município de Fortaleza/CE. A população do estudo englobou os alunos que realizavam seus treinos na academia CB Fitness. Foram selecionados, para compor amostra, 30 voluntários de acordo com os critérios de inclusão e exclusão do estudo. $\mathrm{N}=30$ corresponde a $50 \%$ da população que se enquadrava nos critérios de inclusão estabelecidos no estudo.

\subsection{Critérios de inclusão e exclusão do estudo}

Os voluntários foram selecionados a partir dos seguintes critérios previamente estabelecidos: praticar exercício físico orientado por um de um personal trainer formado em Educação Física e registrado no Conselho Regional de Educação Física (CREF); assinar o termo de consentimento livre e esclarecido (TCLE); Ter idade superior a 18 anos completos na data de coleta dos dados. Foram excluídos do estudo: alunos que não residem no município de Fortaleza/CE; alunos cujo número de treinos semanais seja inferior a duas sessões.

\subsection{Instrumento de pesquisa}

A coleta de dados foi realizada por meio de um questionário com cinco perguntas fechadas, tratando a respeito das características gerais e do treinamento dos entrevistados como: idade; gênero; quantidade de treinos semanal; tempo de treino com personal trainer; e o fator determinante no momento da contratação do profissional. 


\subsection{Aspectos éticos}

No que refere aos aspectos éticos, o questionário não teve perguntas que identificassem os indivíduos e/ou que lhes pudessem causar constrangimento ao responder. Foram incluídos no estudo indivíduos que aceitaram participar voluntariamente da pesquisa, após obtenção de consentimento verbal e uma autorização por escrito, através da assinatura do TCLE. O protocolo do estudo foi realizado de acordo com a Resolução n. ${ }^{\circ} 466 / 12$ do Conselho Nacional de Saúde (CNS), que estabelece as diretrizes e normas regulamentadoras de pesquisas envolvendo seres humanos, e teve seu projeto de pesquisa aprovado pelo comitê de ética local (COMEPE), sob o parecer: ${ }^{\circ} 250 / 16$.

\subsection{Análise e apresentação dos resultados}

Os resultados foram tabulados por meio de uma planilha eletrônica do software Microsoft Office Excel 2011. Em seguida, foram calculados os percentuais de respostas para as questões constantes no questionário, e posteriormente os resultados foram apresentados através da construção de tabelas, que permitiram a descrição e análise dos dados obtidos em relação aos objetivos do estudo.

\section{Resultados e Discussão}

A Tabela 1 mostra a caracterização geral da amostra quanto à idade, ao gênero e ao treinamento com personal trainer.

Tabela 1: Caracterização geral da amostra.

\begin{tabular}{|c|c|c|}
\hline $\begin{array}{l}\text { Variável } \\
\text { Indivíduos }\end{array}$ & $\begin{array}{c}\text { Amostra } \\
30\end{array}$ & $\begin{array}{r}\text { Total } \\
100 \%\end{array}$ \\
\hline $\begin{array}{ll}\text { Idade } & \text { 18-24 anos } \rightarrow \\
& \text { 25-31 anos } \rightarrow \\
& 32-38 \text { anos } \rightarrow \\
& \text { 39-45 anos } \rightarrow \\
\end{array}$ & $\begin{array}{r}10 \\
10 \\
5 \\
5 \\
\end{array}$ & $\begin{array}{l}33 \% \\
33 \% \\
17 \% \\
17 \% \\
\end{array}$ \\
\hline $\begin{array}{ll}\text { Gênero/sexo } & \begin{array}{l}\text { Masculino } \rightarrow \\
\text { Feminino } \rightarrow\end{array}\end{array}$ & $\begin{array}{r}7 \\
23\end{array}$ & $\begin{array}{l}24 \% \\
76 \%\end{array}$ \\
\hline $\begin{array}{ll}\text { Quantidade } & 2 \mathrm{x} \text { semana } \rightarrow \\
\text { de treinos } & 3 \mathrm{x} \text { semana } \rightarrow \\
\text { por } & 4 \mathrm{x} \text { semana } \rightarrow \\
\text { semana } & +4 \mathrm{x} \text { semana } \rightarrow \\
\end{array}$ & $\begin{array}{r}4 \\
13 \\
5 \\
8\end{array}$ & $\begin{array}{c}14 \% \\
42 \% \\
17 \% \\
27 \% \\
\end{array}$ \\
\hline
\end{tabular}




\begin{tabular}{lrrr}
\hline Tempo & 3 meses $\rightarrow$ & 4 & $14 \%$ \\
de treino & 6 meses $\rightarrow$ & 2 & $6 \%$ \\
com & 12 meses $\rightarrow$ & 4 & $14 \%$ \\
personal +12 meses $\rightarrow$ & 20 & $66 \%$ \\
\hline
\end{tabular}

Fonte: Autores

Tendo a caracterização da amostra sido apresentada na tabela anterior, fez-se importante para o estudo conhecer os fatores determinantes no momento da contratação do personal trainer, os resultados obtidos para essa questão foram apresentados abaixo na Tabela 2.

Tabela 2: Fatores determinantes na contratação do personal trainer.

\begin{tabular}{lcc}
\hline Variável & Amostra & Total \\
Indivíduos & 30 & $100 \%$ \\
& & $20 \%$ \\
\hline Formação & 6 & $24 \%$ \\
\hline $\begin{array}{l}\text { Indicação } \\
\text { Metodologia de }\end{array}$ & 7 & \\
Trabalho & 15 & $50 \%$ \\
\hline $\begin{array}{l}\text { Compatibilidade de } \\
\text { horários }\end{array}$ & 2 & $6 \%$
\end{tabular}

\section{Fonte: Autores}

Os resultados mostraram uma relativa distribuição nas idades que consomem os serviços de personal trainer na cidade de acordo com as faixas etárias propostas no estudo. Os resultados achados corroboram os encontrados nos estudos conduzidos por Aguiar, Soares e Guimarães (2008); Sombrio (2011); Mina et al. (2012).

Os resultados obtidos e ilustrados na Tabela 1 revelaram aspectos relevantes. Pode-se observar uma prevalência do público feminino em relação ao masculino, o que mostrou uma tendência maior por parte de mulheres a consumir o serviço de treinamento personalizado. Os resultados obtidos foram semelhantes aos encontros nos estudos realizados por Guimarães (2016), em que 60\% dos entrevistados foram mulheres.

Dados como esses evidenciam que as mulheres tendem, em geral, a consumir mais serviços voltados à saúde. Condongo et al. (2015) compararam os gastos com serviços de atenção básica à saúde 
entre homens e mulheres no estado de São Paulo, e os resultados mostraram que as mulheres têm um maior investimento em saúde em relação aos homens, através da realização de exames e consultas médicas.

Em relação à frequência de treinamento, a resposta mais apontada pelos entrevistados foi 3 vezes por semana. Esses resultados assemelham-se aos achados em outros estudos envolvendo a prática de treinamento personalizado, como os de Jesus e Silva (2008); Azambuja e Santos (2006); Rodrigues e Brandão (2017).

Os resultados apontaram que a maioria dos voluntários utiliza o serviço de treinamento personalizado há mais de 1 ano (66\%), o que nos permite supor uma satisfação com esse tipo de serviço. Esses resultados corroboram os achados por Sombrio (2011); Rodrigues e Brandão (2017), em que os praticantes em sua maioria usufruíam do serviço por um período superior a 12 meses.

A qualidade do serviço prestado parece ser o fator decisivo para o consumo de serviços em gerais pela população. Afinal, o consumidor busca os melhores serviços em busca de resolver as suas necessidades. Isso também foi evidenciado em nosso estudo, na medida em que $50 \%$ dos entrevistados apontaram como fator decisivo para a contratação do personal trainer, a sua metodologia de trabalho utilizada.

A qualidade de serviço refletida através da metodologia de trabalho empregada por meio do treinamento está diretamente associada à motivação e a obtenção de resultados satisfatórios, o que pode refletir na adesão do aluno ao treinamento. No estudo realizado por Rodrigues e Dos Santos (2016) 100\% $(\mathrm{N}=35)$ da amostra que fez parte da pesquisa declarou que os resultados satisfatórios motivam muito ou muitíssimo nos treinamentos. Souto e colaboradores (2010) avaliaram fatores ligados ao cancelamento do serviço de personal trainer em 22 voluntários, sendo 12 homens e 10 mulheres, com idade entre 24 e 70 anos e descobriram que a falta de resultados, a queda na qualidade dos serviços e o preço foram os motivos mais citados como promotores de desistência ao treinamento.

A supervisão por parte de um personal trainer pode trazer realmente melhores resultados através da metodologia diferenciada em comparação com intervenções tradicionais, onde o professor da sala de treinamento atende vários alunos concomitantemente. Segundo Gentil e Bottaro (2010), é possível obter melhores desempenhos em treinamentos com acompanhamento individualizado em comparação com abordagens com menor supervisão por parte do professor.

De acordo com os dados apresentados na Tabela 2, os entrevistados citaram como bastante relevantes para a contratação do personal trainer questões como indicação de amigos/colegas/conhecidos 
(24\%) e a formação do profissional (20\%). Anversa e Oliveira (2011) verificaram através de entrevistas e questionários aplicados com gestores de clínicas e academias da cidade de Maringá-PR que a competência mais importante no momento da contratação do profissional foi a formação e o domínio sobre os conhecimentos técnicos, como podem ser encontrados em $80 \%$ das respostas dos gestores entrevistados.

A competência na elaboração do programa de treinamento, mas também aspectos motivacionais e humanísticos parecem fazer parte de uma metodologia de sucesso para o personal trainer. Em estudo conduzido por Stefanini (2008), realizado com 20 clientes de personal trainer, de ambos os sexos, e com idades entre 25 e 80 anos, buscou-se saber quais as principais características que um personal trainer deve buscar possuir. Os resultados mostraram que as características consideradas como principais pelos clientes foram competência, atenção e bom humor. Ainda nessa pesquisa, foi perguntado aos participantes o que os desmotivava à prática dos seus treinos, e, como resultados, foram apontados pelos clientes aulas e exercícios repetitivos, falta de atenção e desmotivação do próprio personal trainer.

\section{Conclusão}

Através da realização do estudo, procurou-se conhecer os fatores determinantes na contratação de um personal trainer em uma academia de Fortaleza-CE, e de acordo com os resultados obtidos pode-se concluir que a amostra participante do estudo caracteriza-se como sendo formada em sua maioria por jovens adultas, e que majoritariamente treinam com acompanhamento personalizado 3x por semana e utilizam o serviço há mais de 12 meses, o que possivelmente pode evidenciar uma satisfação com o serviço de treinamento personalizado.

Pode-se verificar ainda que a metodologia de trabalho do profissional foi o principal fator apontado pelos entrevistados como determinante na contratação do personal trainer. Esse fato pode refletir o nível cada vez maior de exigência na qualificação profissional por parte dos clientes que se propõem a utilizar o serviço. Observou-se também que além do conhecimento técnico, faz-se importante que o profissional procure desenvolver habilidades relacionadas aos tratos humanísticos e interpessoais dentro de uma metodologia de sucesso nessa profissão. 


\section{Referências}

AguiAR, D. L.; SOARES, A.; GUIMARÃES, A. C. A. Personal Trainer e o Idoso. Fitness Performance Journal. p. 185-94, 2008.

ANVERSA, A. L. B.; OLIVEIRA, A. A. B. Personal Trainer: Competências profissionais demandadas pelo mercado de trabalho. Pensar a Prática, Goiânia, v. 14, n. 3, p. 117, 2011.

AZAMBUJA, C. R.; DOS SANTOS, D. L. Consumo de Recursos Ergogênicos e Farmacológicos por Praticantes de Musculação das Academias de Santa Maria-RS. Revista Brasileira de Fisiologia do Exercício, São Paulo, v. 5, n. 1, p.27-33, 2006.

BERESFORD, H.; SILVA, I. L.; GARAY, L. C. O treinamento personalizado: um enfoque paradigmático da performance para o bem estar. Revista Eletrônica da Escola de Educação Física e Desportos, UFRJ, v. 4, n. 1, 2008.

CONDONGO, J. S.; TURI, B. C.; FERNANDES, S. A.; MONTEIRO, H. L. Comparação de gastos com serviços de atenção básica à saúde de homens e mulheres em Bauru. Epidemiologia Serviço e Saúde, Brasília, v.24, n.1, 2015.

FREITAS, C. M. S. M.; SANTIAGO, M. S.; VIANA, A. T.; LEÃO, A. C.; FREYRE, C. Aspectos motivacionais que influenciam a adesão e manutenção de idosos a programas de exercícios físicos. Revista Brasileira de Cineantropometria \& Desempenho Humano. v. 9, n. 1, p.92-100, 2007.

GENTIL, P.; BOTTARO, M. Influence of supervision ratio on muscle adaptations to resistance training in non trained subjects. Journal of Strength and Conditioning Research, 2010.

GUIMARÃES, J. A. Perfil de aderência e permanência de alunos de personal trainer da cidade de Cuiabá. Revista Brasileira de Nutrição Esportiva, São Paulo, v. 6, n. 33, 2p. 241-245, 2012.

JESUS, E. V.; SILVA, M. D. B. Suplemento alimentar como recurso ergogênico por praticantes de musculação em academias. ANAIS do III Encontro de Educação Física e Áreas Afins Núcleo de Estudo e Pesquisa em Educação Física (NEPEF)/Departamento de Educação Física/UFPI, 2008.

LIBERALI, R. Metodologia Científica Prática: um saber fazer competente da saúde à educação. Florianópolis, 2008.

MINA, D. S. et al. Group Exercise versus Personal Training for Prostate Cancer Patients: A Pilot Randomized Trial. Journal of Cancer Therapy, p. 146-156, 2012.

PRADO, A. O. V.; LIBERALI, R. Motivos que levam mulheres a procurarem treinamento personalizado. Revista Brasileira de Prescrição e Fisiologia do Exercício, São Paulo, v. 2, n. 11, p. 565, 2008.

RODRIGUES, A. L. P.; BRANDÃO, D. C. Fatores motivacionais que levam mulheres entre 18 e 30 anos a procura e adesão ao treinamento com personal trainer em uma academia na cidade de Fortaleza-CE. Revista Brasileira de Nutrição Esportiva, São Paulo, v. 11, n. 62, p.168-175, 2007. 
RODRIGUES, A. L. P.; Dos Santos, R. V. Aspectos motivacionais para a prática de musculação entre jovens de 18 a 25 anos do município de Fortaleza-CE. Revista Brasileira de Prescrição e Fisiologia do Exercício, São Paulo, v. 10, n. 58, p. 308-313, 2016.

SANTOS, S. C.; KNIJNIK, J. D. Motivos de adesão à prática de atividade física na vida adulta intermediária. Revista Mackenzie de Educação Física e Esporte, São Paulo, v. 5, n. 1, p.25, 2006.

SOMBRIO, D. A. Motivos de adesão e aderência a prática de exercício físico do cliente/aluno de personal trainer no município de Criciúma-SC. Trabalho de Conclusão de Curso. Universidade do Extremo Sul Catarinense, UNESC, 2011

SOUTO, R. C. M.; DINARDI, R. R.; RIBEIRO, J. R. C.; ANDRADE, A. G. P. Aspectos envolvidos na contratação e cancelamento de um personal trainer. Coleção Pesquisa em Educação Física. São Paulo, v. 9, n. 4, 2010.

STEFANINI, F. A Psicologia interferindo na motivação: uma ferramenta essencial para o sucesso de um personal trainer. Trabalho de Conclusão de Curso. Universidade Estadual Paulista Júlio de Mesquita Filho, 2008

TAHARA, A. K.; SILVA, K. A. A prática de exercícios Físicos na promoção de um estilo de vida ativo. Revista Digital Buenos Aires, Buenos Aires, v. 9, n.61, 2003.

\section{Como citar este artigo}

RODRIGUES, A. L. P. Fatores determinantes na contratação de um personal trainer em Fortaleza/CE. Revista Kinesis, Santa Maria, v.38, p.01-09, 2020.

* O presente trabalho não contou com apoio financeiro de nenhuma natureza para sua realização. 